\title{
Avaliação do Estado Nutricional e Capacidade Funcional de Pacientes Oncológicos em Quimioterapia de Caxias do Sul - RS
}

https://doi.org/10.32635/2176-9745.RBC.2020v66n2.377

\author{
Evaluation of Nutritional Status and Functional Capacity in Oncologic Patients undergoing Chemotherapy in Caxias do Sul - RS \\ Evaluación del Estado Nutricional y Capacidad Funcional en Pacientes Oncológicos en Quimioterapia de Caxias do Sul - RS
}

Gabriela Argenta Isoton'; Caroline da Silva Scotti ${ }^{2}$; Joana Zanotti ${ }^{3}$

Resumo

Introduçáo: Câncer é o nome dado a um conjunto de doenças que têm em comum o crescimento desordenado de células. O estado nutricional debilitado é frequente em pacientes oncológicos e interfere de forma negativa nas habilidades de vida diárias do paciente. Objetivo: Avaliar o estado nutricional e a capacidade funcional de pacientes oncológicos em quimioterapia. Método: Estudo epidemiológico observacional com delineamento transversal, composto por uma amostra obtida por conveniência, de 213 indivíduos em tratamento quimioterápico ambulatorial. Os entrevistados responderam à avaliação subjetiva global produzida pelo paciente (ASG-PPP); a capacidade funcional foi verificada por meio da força de preensão palmar (FPP) e da escala de performance status Eastern Cooperative Oncology Group (ECOG). Resultados: Foram avaliados 213 pacientes, 56,3\% idosos e 54,0\% do sexo feminino. Com exceção dos indivíduos com neoplasia de cabeça e pescoço, observou-se maior prevalência de bem nutridos conforme a ASG-PPP ( $\mathrm{p}=0,004)$; em relação ao índice de massa corporal (IMC), exceto para indivíduos com câncer de mama, a maior prevalência foi de eutrofia ( $\mathrm{p}=0,010)$. Os indivíduos com FPP adequada $(54,9 \%)$ apresentaram-se bem nutridos conforme a ASG $(91,5 \%)$ ( $\mathrm{p} \leq 0,0001)$ e sobrepeso de acordo com o IMC (46,2\%) $(\mathrm{p}=0,010)$. Ainda, os indivíduos totalmente ativos $(69,0 \%)$ eram bem nutridos conforme a ASG $(89,8 \%)(\mathrm{p} \leq 0,0001)$ e os eutróficos segundo o IMC $(44,2 \%)$ ( $\mathrm{p}=0,003)$, demonstrando diferença significativa na avaliação do estado nutricional com a capacidade funcional. Conclusáo: $\mathrm{O}$ estudo demonstrou que a maioria dos pacientes oncológicos em quimioterapia tinham satisfatório estado nutricional, adequada força muscular e eram totalmente ativos.

Palavras-chave: Estado Nutricional; Força Muscular; Neoplasias/tratamento farmacológico.

\begin{abstract}
Introduction: Cancer is the name given to a set of diseases that have in common the disorderly growth of cells. Impaired nutritional status is frequent in oncologic patients and negatively interferes in the patient's daily life abilities. Objective: To evaluate the nutritional status and functional capacity of oncologic patients undergoing chemotherapy treatment. Method: Cross-sectional design, observational epidemiological study, with a sample obtained by convenience from 213 individuals undergoing outpatient chemotherapy treatment. The interviewees answered to the Patient-Generated Subjective Global Assessment (PG-SGA); functional ability was verified using handgrip strength (HGS) (hand dynamometry) and through the Eastern Cooperative Oncology Group (ECOG) scale for performance scale. Results: 213 patients were evaluated, $56.3 \%$, elderly and $54.0 \%$, females. Excluding head and neck neoplasms, there was a higher prevalence of well-nourished individuals according to PG-SGA ( $\mathrm{p}=0.004)$; in relation to the body mass index (BMI), except for individuals with breast cancer, the highest prevalence was eutrophy $(\mathrm{p}=0.010)$. Individuals with adequate HGS (54.9\%) were well nourished according to the SGA (91.5\%) $(\mathrm{p} \leq 0.0001)$ and overweight according to the BMI $(46.2 \%)(\mathrm{p}=0.010)$. Still, fully active individuals $(69.0 \%)$, were well nourished according to SGA $(89.8 \%)(\mathrm{p} \leq 0.0001)$ and eutrophic according to BMI $(44.2 \%)(\mathrm{p}=0.003)$, showing a significant difference in the assessment of nutritional status with functional capacity. Conclusion: The study demonstrated that the majority of cancer patients undergoing chemotherapy had satisfactory nutritional status, adequate muscle strength and were fully active.
\end{abstract}

Key words: Nutritional Status; Muscle Strength; Neoplasms/drug therapy.

\section{Resumen}

Introducción: Cáncer es el nombre dado a un conjunto de enfermedad que tienen en común el crecimiento celular desordenado. El estado nutricional débil es frecuente en pacientes oncológicos e interfiere negativamente con las habilidades de la vida diaria del paciente. Objetivo: Evaluar el estado nutricional y capacidad funcional de los pacientes oncológicos en quimioterapia. Método: Estudio epidemiológico observacional con delineamiento transversal, compuesto por muestra obtenida por conveniencia, 213 individuos en tratamiento ambulatorial. Los entrevistados respondieron a la evaluación subjetiva global producida por el paciente (ESG-PPP); la capacidad funcional se verificó utilizando la fuerza de la empuñadura (FPP) y a través de la escala de rendimiento del Eastern Cooperative Oncology Group (ECOG). Resultados: Se evaluaron 213 pacientes, 56,3\%ancianos y 54,0\% mujeres. Con excepción de las neoplasias de cabeza y cuello, hubo una mayor prevalencia de individuos bien nutridos según el ESG-PPP ( $p=0,004)$; en relación con el índice de masa corporal (IMC), a excepción de las personas con cáncer de mama, la prevalencia más alta fue la eutrofia $(\mathrm{p}=0,010)$. Las personas con FPP adecuada $(54.9 \%)$ estaban bien nutridas según la ESG $(91,5 \%)(\mathrm{p} \leq 0,0001)$ y con sobrepeso con el IMC (46,2\%) ( $\mathrm{p}=0,010)$. Aun así, los individuos completamente activos $(69,0 \%)$ estaban bien nutridos según ESG $(89,8 \%)(p \leq 0,0001)$ y eutróficos según el IMC (44,2\%) ( $\mathrm{p}=0,003)$, mostrando una diferencia significativa en la evaluación del diagnóstico nutricional con capacidad funcional. Conclusión: El estudio demostró que la mayoría de los pacientes con cáncer que reciben quimioterapia con un estado nutricional satisfactorio, una fuerza muscular adecuada y completamente activa.

Palabras clave: Estado Nutricional; Fuerza Muscular; Neoplasias/ tratamiento farmacológico. 


\section{INTRODUÇÃO}

O câncer é caracterizado pelo conjunto de mais de 100 doenças, nas quais ocorre desenvolvimento irregular e desordenado de células ${ }^{1}$. A estimativa mundial mostra que, em 2018, ocorreram 18,1 milhóes de novos casos de câncer e 9,5 milhóes de óbitos². Dados do Instituto Nacional de Câncer José de Alencar Gomes da Silva (INCA) estimam, para o triênio 2020-2022, a ocorrência de 625 mil novos casos de câncer no Brasil. A distribuição da incidência por Região geográfica demostra que a Região Sudeste concentra mais de $60 \%$ da incidência, seguida pelas Regióes Nordeste $(27,8 \%)$ e Sul do país $(23,4 \%)^{3}$.

A redução de peso corporal e a desnutrição são consideradas os principais distúrbios nutricionais no paciente oncológico, decorrentes das alteraçóes metabólicas causadas pela neoplasia, do aumento na demanda energética promovido pelo tumor e tratamento da doença ${ }^{4}$. O tratamento antitumoral envolve cirurgia, quimioterapia e radioterapia e, entre as modalidades de tratamento, destaca-se a quimioterapia, que é um método de maior escolha para diminuir a chance do crescimento desordenado das células, por meio de uma terapêutica que usa drogas isoladas ou combinadas, a fim de evitar que a doença progrida ou de promover a cura 5 .

Em razão do estado nutricional debilitado, o paciente pode apresentar uma queda da funcionalidade, uma vez que a desnutrição calórico-proteica afeta diretamente a perda de fibras musculares esqueléticas e, consequentemente, a diminuição da força muscular ${ }^{6}$. A funcionalidade pode ser compreendida como a capacidade de o indivíduo efetuar determinadas atividades ou funçóes que influenciam nos comportamentos simples e complexos exigidos em seu dia a dia. O comprometimento funcional incapacita o indivíduo para realização de atividades básicas, como cuidar de si mesmo e de seu entorno de forma independente ${ }^{7}$. Em geral, pacientes com câncer têm alteração da capacidade funcional durante a trajetória da doençą ${ }^{8}$.

Portanto, este estudo teve como objetivo avaliar o estado nutricional e a capacidade funcional de pacientes oncológicos em quimioterapia de Caxias do Sul - RS.

\section{MÉTODO}

Foi realizado um estudo epidemiológico observacional com delineamento transversal, com amostra obtida por conveniência, coletada no período de janeiro a fevereiro de 2019, em um Hospital Escola na cidade de Caxias do Sul - RS. Foram incluídos no estudo indivíduos de ambos os sexos, com idade superior a 19 anos, independentemente do tempo de diagnóstico e ciclo do tratamento quimioterápico ambulatorial. Excluíram-se os pacientes que possuíam alguma deficiência intelectual e motora, gestantes, puérperas e os que não obtinham a avaliação da qualidade de vida descrita no prontuário do paciente.

Os dados foram coletados após aprovação do Comitê de Ética em Pesquisa de ambas as instituiçôes envolvidas, sob os números de parecer 2.571.056 e 2.726.138. A pesquisa faz parte de um projeto guarda-chuva intitulado: Risco nutricional e sintomas associados à localização do tumor em pacientes oncológicos em tratamento quimioterápico. Todos os procedimentos foram seguidos de acordo com a Resolução número $466^{\circ}$ e todos os pacientes foram informados sobre o objetivo do estudo e a confidencialidade dos dados, sendo assinado e entregue o Termo de Consentimento Livre e Esclarecido (TCLE).

Foi aplicada a avaliação subjetiva global produzida pelo paciente (ASG-PPP), que consiste em um questionário autoaplicativo, validado e traduzido ${ }^{10}$, dividido em duas partes. $\mathrm{Na}$ primeira, o paciente responde, descrevendo sua alteração de peso, da ingestáo alimentar, sintomas relacionados ao câncer e alteraçóes da capacidade funcional. Na segunda parte, respondida pelo profissional que aplica o questionário, as questôes são baseadas nos fatores associados ao diagnóstico, que aumentam a demanda metabólica. Ao final da avaliação, o paciente pode ser classificado como A (bem nutrido), B (suspeita ou desnutrição moderada) ou C (gravemente desnutrido).

Os dados de peso e estatura para a avaliação do índice de massa corporal (IMC) foram obtidos com base nas respostas da ASG-PPP e classificados de acordo com os padrôes da Organização Mundial da Saúde (OMS) e do Ministério da Saúde. A classificação do IMC do adulto está dividida em: baixo peso $\leq 18,5 \mathrm{~kg} / \mathrm{m}^{2}$; peso adequado $>18,5$ até $24,9 \mathrm{~kg} / \mathrm{m}^{2}$; sobrepeso $\geq 25$ até $29,9 \mathrm{~kg} / \mathrm{m}^{2}$; e obesidade $\geq 30,0 \mathrm{~kg} / \mathrm{m}^{2}$. Para indivíduos idosos, a classificação utilizada foi a seguinte: baixo peso $\leq 22 \mathrm{~kg} / \mathrm{m}^{2}$; peso adequado $>22$ até $<27 \mathrm{~kg} / \mathrm{m}^{2}$; e sobrepeso $\geq 27 \mathrm{~kg} / \mathrm{m}^{211,12}$.

Informaçóes sobre capacidade funcional foram coletadas de duas maneiras, primeiramente pela escala desenvolvida pelo Eastern Cooperative Oncology Group (EGOC), publicada em 1982, a qual avalia o performance status, preenchida pelos enfermeiros do ambulatório e descrita no prontuário do paciente. Essa escala estabelece escores de classificação de 0 a $4^{13}$. Conforme descrito a seguir.

Escore 0: corresponde ao paciente totalmente ativo, capaz de realizar todo o desempenho pré-doença sem restrição. Escore 1: restrição a atividades físicas rigorosas (é capaz de trabalhos leves e de natureza sedentária). Escore 2: capaz de realizar todos os autocuidados, mas 
incapaz de realizar qualquer atividade de trabalho (em pé aproximadamente $50 \%$ das horas em que o paciente está acordado). Escore 3: capaz de realizar somente autocuidados limitados, confinado ao leito ou à cadeira mais de 50\% das horas em que o paciente está acordado. Escore 4: completamente incapaz de realizar autocuidados básicos, totalmente confinado ao leito ou à cadeira ${ }^{13}$.

O outro método utilizado para avaliação da capacidade funcional, foi o teste de força de preensão palmar (FPP) realizado por meio da dinamometria manual, que visa a estimar o estado funcional do músculo esquelético, fazendo uso do dinamômetro hidráulico da marca SAEHAN ${ }^{\circ}$. Os pacientes executaram o teste em posição sentada, com o cotovelo flexionado em ângulo de $90^{\circ}$, antebraço e pulso em posição neutra. Os sujeitos realizaram três contraçôes isométricas máximas, com uma breve pausa entre as medidas. Após obtidas as três medidas de cada mão (dominante e não dominante), foram somadas as medidas e divididas por três, e assim se obteve uma medida média. $\mathrm{O}$ valor de referência da FPP, considerado adequado para mulheres, segundo o
European Working Group on Sarcopenia in Older People (EWGSOP) ${ }^{14}$, é $\geq 16 \mathrm{~kg} / \mathrm{f}$ e, para os homens, $\geq 27 \mathrm{~kg} / \mathrm{f}^{14}$. Considerou-se fraqueza muscular valores inferiores.

Os dados foram tabulados e analisados no programa Statistical Package for the Social Sciences (SPSS Statistic Data) versão 23.0; e aplicado o teste qui-quadrado de Pearson para avaliar a diferença nas proporçóes de desfechos categóricos, sendo considerado um nível de significância de $5 \%(\mathrm{p}<0,05)$.

\section{RESULTADOS}

Foram avaliados 213 pacientes, classificados em adultos (19 a 59 anos) e idosos (acima de 60 anos), sendo $43,7 \%$ adultos e $56,3 \%$ idosos e a maioria do sexo feminino $(54,0 \%)$. Na Tabela 1 , são apresentadas as variáveis demográficas, clínicas e de funcionalidade em relação ao diagnóstico nutricional da ASG-PPP.

Ainda na Tabela 1 , verificou-se associação estatisticamente significativa entre a localização do tumor e a classificação da ASG-PPP ( $p=0,004)$, sendo a maior

Tabela 1. Descrição das variáveis demográficas, clínicas e de funcionalidade em relação à ASG-PPP em indivíduos em tratamento oncológico em quimioterapia, $2019(\mathrm{n}=213)$

\begin{tabular}{|c|c|c|c|c|c|}
\hline \multirow[b]{2}{*}{ Variáveis } & \multirow[b]{2}{*}{ n (\%) } & \multicolumn{3}{|c|}{ RP ASG-PPP } & \multirow[b]{2}{*}{ p-valor* } \\
\hline & & $\begin{array}{c}\text { Bem nutrido } \\
(n=160) 75,2 \%\end{array}$ & $\begin{array}{c}\text { Desnutrido } \\
\text { moderado } \\
(n=45) 21,1 \%\end{array}$ & $\begin{array}{c}\text { Gravemente } \\
\text { desnutrido } \\
(n=8) 3,7 \%\end{array}$ & \\
\hline Idade & & & & & 0,005 \\
\hline Adulto & $93(43,7)$ & $80(86,0)$ & $12(12,9)$ & $1(1,1)$ & \\
\hline Idoso & $120(56,3)$ & $80(66,7)$ & $33(27,5)$ & $7(5,8)$ & \\
\hline Sexo & & & & & 0,371 \\
\hline Masculino & $98(46,0)$ & $73(74,5)$ & $23(23,5)$ & $2(2,0)$ & \\
\hline Feminino & $115(54,0)$ & $87(75,7)$ & $22(19,1)$ & $6(5,2)$ & \\
\hline Diagnóstico de câncer & & & & & 0,004 \\
\hline Próstata & $15(7,0)$ & $10(66,7)$ & $4(26,7)$ & $1(6,7)$ & \\
\hline Pulmão & $18(8,5)$ & $11(61,1)$ & $5(27,8)$ & $2(11,1)$ & \\
\hline Hematológico & $61(28,6)$ & $46(75,4)$ & $15(24,6)$ & $0(0,0)$ & \\
\hline Mama & $31(14,6)$ & $24(77,4)$ & $6(19,4)$ & $1(3,2)$ & \\
\hline $\mathrm{TGI}^{*}$ & $31(14,6)$ & $28(90,3)$ & $2(6,5)$ & $1(3,2)$ & \\
\hline Cabeça e pescoço & $12(5,6)$ & $4(33,3)$ & $5(41,7)$ & $3(25,0)$ & \\
\hline Outros & $45(21,1)$ & $37(82,2)$ & $8(17,7)$ & $0(0,0)$ & \\
\hline FPP* & & & & & $\leq 0,0001$ \\
\hline Adequado & $117(54,9)$ & $107(91,5)$ & $9(7,7)$ & $1(0,9)$ & \\
\hline Fraqueza muscular & $96(45,1)$ & $53(55,2)$ & $36(37,5)$ & $7(7,3)$ & \\
\hline ECOG ${ }^{\epsilon}$ & & & & & $\leq 0,0001$ \\
\hline Escore 0 & $147(69,0)$ & $132(89,8)$ & $14(9,5)$ & $1(0,7)$ & \\
\hline Escore 1 & $47(22,1)$ & $21(44,7)$ & $24(51,1)$ & $2(4,3)$ & \\
\hline Escore 2 & $15(7,0)$ & $5(33,3)$ & $6(40,0)$ & $4(26,7)$ & \\
\hline Escore 3 & $4(1,9)$ & $2(50,0)$ & $1(25,0)$ & $1(25,0)$ & \\
\hline
\end{tabular}

Legendas: ^RP ASG-PPP: razão de prevalência de avaliação subjetiva global produzida pelo paciente; FPP: força de preensão palmar; ${ }^{\epsilon}$ ECOG: Eastern Cooperative

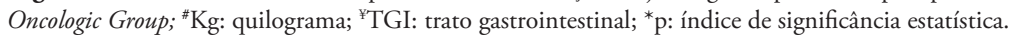

Nota: Teste qui-quadrado de Pearson para heterogeneidade. Valores em negrito são estatisticamente significativos ( $\mathrm{p} \leq 0,05)$. 
prevalência de indivíduos diagnosticados com câncer hematológico (28,6\%). Em relação à ASG-PPP, 90,3\% dos indivíduos com neoplasias do trato gastrointestinal (TGI) foram classificados como bem nutridos e $41,7 \%$ dos entrevistados com neoplasias de cabeça e pescoço, como desnutridos moderados.

De acordo com a FPP, 54,9\% dos indivíduos avaliados no estudo apresentaram força adequada e, destes, $91,5 \%$ foram classificados como bem nutridos. Dos pacientes com fraqueza muscular, 55,2\% foram classificados como bem nutridos. Foi verificada associação estatisticamente significante entre a FPP e a classificaçáo da ASG-PPP $(\mathrm{p} \leq 0,0001)$.

Ao analisar o ECOG com a classificação da ASG-PPP, também foi verificada associação estatisticamente significante $(\mathrm{p} \leq 0,0001)$, sendo que, dos pacientes totalmente ativos (escore 0), 89,8\% foram classificados como bem nutridos e, entre os pacientes com restrição a atividades físicas rigorosas (escore 1), 51,1\% foram classificados em desnutridos moderados (Tabela 1).

$\mathrm{Na}$ Tabela 2, são apresentadas as variáveis demográficas, clínicas e de funcionalidade em relação ao IMC. De acordo com o IMC e a idade dos investigados, 45,8\% dos idosos apresentaram peso adequado e $41,9 \%$ dos adultos apresentaram sobrepeso $(\mathrm{p} \leq 0,0001)$. Em relação ao sexo, $52 \%$ dos homens apresentaram peso adequado e $43,5 \%$ das mulheres, sobrepeso ( $\mathrm{p}=0,041)$. Ao analisar a localização do tumor e a classificação do IMC, 58,1\% dos indivíduos que possuíam diagnóstico de câncer de TGI apresentaram eutrofia e $41,9 \%$ dos entrevistados

Tabela 2. Descrição das variáveis demográficas, clínicas e de funcionalidade em relação ao IMC em indivíduos em tratamento oncológico em quimioterapia, $2019(n=213)$

\begin{tabular}{|c|c|c|c|c|c|c|}
\hline \multirow[b]{2}{*}{ Variáveis } & \multirow[b]{2}{*}{ n (\%) } & \multicolumn{4}{|c|}{ RP IMC } & \multirow[b]{2}{*}{ p-valor* } \\
\hline & & $\begin{array}{c}\text { Baixo peso } \\
\begin{array}{c}(n=23) \\
10,8 \%\end{array}\end{array}$ & $\begin{array}{c}\text { Peso } \\
\text { adequado } \\
\begin{array}{c}(n=89) \\
41,8 \%\end{array}\end{array}$ & $\begin{array}{c}\text { Sobrepeso } \\
\qquad \begin{array}{c}(n=83) \\
39,0 \%\end{array}\end{array}$ & $\begin{array}{l}\text { Obesidade } \\
\qquad \begin{array}{c}(n=18) \\
8,5 \%\end{array}\end{array}$ & \\
\hline Idade & & & & & & $\leq 0,0001$ \\
\hline Adulto & $93(43,7)$ & $2(2,2)$ & $34(36,6)$ & $39(41,9)$ & $18(19,4)$ & \\
\hline Idoso & $120(56,3)$ & $21(17,5)$ & $55(45,8)$ & $44(36,7)$ & $0(0,0)$ & \\
\hline Sexo & & & & & & 0,041 \\
\hline Masculino & $98(46,0)$ & $8(8,2)$ & $51(52,0)$ & $33(33,7)$ & $6(6,1)$ & \\
\hline Feminino & $115(54,0)$ & $15(13,0)$ & $38(33,0)$ & $50(43,5)$ & $12(10,4)$ & \\
\hline Diagnóstico & & & & & & 0,010 \\
\hline Próstata & $15(7,0)$ & $2(13,0)$ & $7(46,7)$ & $6(40,0)$ & $0(0,0)$ & \\
\hline Pulmão & $18(8,5)$ & $5(27,8)$ & $10(55,6)$ & $3(16,7)$ & $0(0,0)$ & \\
\hline Hematológico & $61(28,6)$ & $6(9,8)$ & $25(41,0)$ & $22(36,1)$ & $8(13,1)$ & \\
\hline Mama & $31(14,6)$ & $2(6,5)$ & $12(38,7)$ & $13(41,9)$ & $4(12,9)$ & \\
\hline $\mathrm{TGI}^{*}$ & $31(14,6)$ & $2(6,5)$ & $18(58,1)$ & $10(32,3)$ & $1(3,2)$ & \\
\hline Cabeça e pescoço & $12(5,6)$ & $4(33,3)$ & $5(41,7)$ & $2(16,7)$ & $1(8,3)$ & \\
\hline Outros & $45(21,1)$ & $2(4,4)$ & $12(26,7)$ & $27(60,0)$ & $4(8,9)$ & \\
\hline FPP* & & & & & & 0,010 \\
\hline Adequado & $117(54,9)$ & $6(5,1)$ & $48(41,0)$ & $54(46,2)$ & $9(7,7)$ & \\
\hline Fraqueza muscular & $96(45,1)$ & $17(17,7)$ & $41(42,7)$ & $29(30,2)$ & $9(9,4)$ & \\
\hline ECOG $€$ & & & & & & 0,003 \\
\hline Escore 0 & $147(69,0)$ & $7(4,8)$ & $65(44,2)$ & $61(41,5)$ & $14(9,5)$ & \\
\hline Escore 1 & $47(22,1)$ & $10(21,3)$ & $20(42,6)$ & $16(34,0)$ & $1(2,1)$ & \\
\hline Escore 2 & $15(7,0)$ & $4(26,7)$ & $4(26,7)$ & $5(33,3)$ & $2(13,3)$ & \\
\hline Escore 3 & $4(1,9)$ & $2(50,0)$ & $0(0,0)$ & $1(25,0)$ & $1(25,0)$ & \\
\hline
\end{tabular}

Legendas: ^RP IMC: razão de prevalência do índice de massa corporal; *FPP: força de preensão palmar; ${ }^{\epsilon}$ ECOG: Eastern Cooperative Oncologic Group; ${ }^{*}$ Kg: quilograma; ¥TGI: trato gastrointestinal; *p: índice de significância estatística.

Nota: Teste qui-quadrado para heterogeneidade. Valores em negrito são estatisticamente significativos $(\mathrm{p} \leq 0,05)$. 
com câncer de mama foram classificados com sobrepeso $(\mathrm{p}=0,010)$ (Tabela 2).

Ainda na Tabela 2, ao comparar a FPP com o IMC, observou-se que $46,2 \%$ dos entrevistados com força adequada foram classificados como sobrepeso e $42,7 \%$ dos que possuíam baixa força, classificados com peso adequado $(\mathrm{p}=0,010)$. Em relação ao EGOC, 44,2\% dos indivíduos totalmente ativos (escore 0 ) apresentaram eutrofia, 34,0\% com restrição de atividades físicas rigorosas (escore 1) foram classificados com sobrepeso e 50,0\% dos que realizaram somente autocuidados limitados (escore 4) apresentaram desnutrição $(\mathrm{p}=0,003)$.

$\mathrm{Na}$ Tabela 3, é apresentada a descrição do IMC em relação à ASG-PPP. De acordo com a classificação do IMC, 75,3\% dos entrevistados com peso adequado apresentaram-se bem nutridos e $23,6 \%$ foram classificados em desnutridos moderados segundo a ASG-PPP. Entre os entrevistados com baixo peso, 56,5\% foram classificados com desnutrição moderada e 30,4\% em gravemente desnutridos conforme a ASG-PPP. Dos 83 indivíduos classificados com sobrepeso, dez (12,0\%) apresentaram desnutrição moderada em relação à avaliação. Foi verificada associação estatisticamente significante entre as variáveis do IMC e da ASG-PPP ( $\mathrm{p} \leq 0,0001)$ (Tabela 3).

\section{DISCUSSÃO}

O presente estudo teve como objetivo avaliar o estado nutricional e a capacidade funcional de pacientes submetidos à quimioterapia. As análises do estudo revelaram a predominância de pacientes idosos $(56,3 \%)$, o que se deve ao fato de o envelhecimento estar associado à maior incidência de câncer, pois, com o avanço da idade, ocorre diminuição da capacidade de reparaçáo celular. A predominância do sexo feminino $(54,0 \%)$ encontrada no presente estudo pode ser explicada por causa da maior expectativa de vida feminina e da maior taxa de mortalidade masculina no Brasil ${ }^{15}$.

Em relação à localização dos tumores, um estudo realizado com 50 pacientes oncológicos de um hospital de Uberlândia - MG apresentou maior prevalência de câncer hematológico (24\%) e TGI (14\%), esses achados corroboram o atual estudo $^{16}$. Segundo dados do INCA, para o triênio 2020-2022, estimam-se, no Brasil, 5.920 casos de leucemia em homens e 4.890 em mulheres. Em relação ao linfoma não Hodgkin, estimam-se 6.580 casos em homens e 5.450 em mulheres. Já as estimativas de novos casos de cânceres do TGI são bem mais elevadas: câncer de cólon e reto 40.990 casos e estômago 21.230, para o mesmo período 3 .

Conforme os resultados da ASG-PPP obtidos neste estudo, a maioria dos indivíduos com as neoplasias hematológicas e de TGI estavam bem nutridos. Resultados contrários ao Inquérito Brasileiro de Nutrição Oncológica $(\mathrm{IBNO})^{17}$, o qual evidência a elevada frequência de desnutrição nos portadores de câncer do TGI e em pacientes com doenças hematológicas malignas, como leucemias e linfomas. Esse fato pode ser justificado em virtude de o IBNO ter avaliado indivíduos em tratamento quimioterápico hospitalar de 45 instituições de 16 Estados e do Distrito Federal, gerando 4.822 avaliaçóes, diferente deste estudo, no qual foram avaliados pacientes em tratamento ambulatorial ${ }^{17}$.

Ainda segundo a ASG-PPP, 41,7 \% dos indivíduos com diagnóstico de câncer de cabeça foram classificados como desnutridos moderados. Achado semelhante ao encontrado por Cunha et al. ${ }^{18}$, no qual $45,5 \%$ dos 576 indivíduos entrevistados apresentaram a mesma localização do tumor e classificação. Gomes e Maio ${ }^{4}$ encontraram resultados parecidos em um estudo realizado em 2015,

Tabela 3. Descrição do IMC em relação à ASG-PPP em indivíduos em tratamento oncológico em quimioterapia, 2019 (n=213)

\begin{tabular}{|c|c|c|c|c|c|}
\hline \multirow[b]{2}{*}{ Variável } & \multirow[b]{2}{*}{ n (\%) } & \multicolumn{3}{|c|}{ RP ASG-PPP } & \multirow[b]{2}{*}{ p-valor* } \\
\hline & & $\begin{array}{c}\text { Bem nutrido } \\
(n=160) \\
75,2 \%\end{array}$ & $\begin{array}{c}\text { Desnutrido } \\
\text { moderado } \\
(n=45) \\
21,1 \%\end{array}$ & $\begin{array}{c}\text { Gravemente } \\
\text { desnutrido } \\
(\mathbf{n = 8}) \\
3,7 \%\end{array}$ & \\
\hline \multicolumn{5}{|l|}{$I M C^{\epsilon}$} & $\leq 0,0001$ \\
\hline Obesidade & $18(8,5)$ & $17(94,4)$ & $1(5,6)$ & $0(0,0)$ & \\
\hline Sobrepeso & $83(39,0)$ & $73(88,0)$ & $10(12,0)$ & $0(0,0)$ & \\
\hline Peso adequado & $89(41,8)$ & $67(75,3)$ & $21(23,6)$ & $1(1,1)$ & \\
\hline Baixo peso & $23(10,8)$ & $3(13,0)$ & $13(56,5)$ & $7(30,4)$ & \\
\hline
\end{tabular}

Legendas: ^RP ASG-PPP: razão de prevalência de avaliação subjetiva global produzida pelo paciente; ${ }^{\epsilon}$ IMC: índice de massa corporal; *p: índice de significância estatística.

Nota: Teste qui-quadrado para heterogeneidade. Valores em negrito são estatisticamente significativos $(\mathrm{p} \leq 0,05)$. 
em que 59,5\% dos pacientes com câncer de cabeça e pescoço em quimioterapia foram classificados com risco nutricional ou desnutrição. É importante salientar que, por conta da localização do tumor, os indivíduos com câncer de cabeça e pescoço podem apresentar disfagia, o que dificulta a ingesta alimentar causando perda de peso a curto prazo ${ }^{4}$.

Dos entrevistados classificados com fraqueza muscular, 55,2\% apresentavam-se bem nutridos conforme a ASG-PPP. Esse achado pode ser relacionado à maior prevalência de mulheres com sobrepeso, neoplasia de mama e idosas. A localização do tumor e os efeitos colaterais do tratamento podem interferir na funcionalidade do paciente; a força muscular tende a ser diferente entre os homens e mulheres, sendo que o sexo feminino apresenta valores inferiores aos do sexo masculino. Em relação à idade, os idosos sofrem um declínio da força, em razão do envelhecimento, associando a baixa FPP com a idade ${ }^{19}$.

Ao avaliar o IMC, o estudo observou que apenas $10,8 \%$ dos pacientes apresentavam déficit nutricional, corroborando os achados de Firnkes et al. ${ }^{20}$, nos quais 7,8\% dos pacientes apresentam IMC menor que $18,5 \mathrm{~kg} / \mathrm{m}^{2}$. Em contraponto, sabe-se que o IMC é um parâmetro que possui valor limitado, pois os avaliados podem apresentar perda de massa magra e aumento da retençáo hídrica, mascarando o real estado nutricional ${ }^{21}$.

Quando comparada a classificação de IMC baixo peso com a FPP, 21 idosos obtiveram baixo peso, sendo que, destes, 17 apresentaram fraqueza muscular. Sabe-se a força muscular é um importante indicativo de saúde global para ambos os sexos e que o envelhecimento acarreta depleção do estado nutricional, causando alteraçôes negativas na capacidade funcional ${ }^{22}$. Portanto, os níveis adequados de força muscular são necessários para que os indivíduos possam realizar todas as tarefas diárias, desde atividades de trabalho até as recreativas ${ }^{19}$.

Neste estudo, houve elevada prevalência de eutrofia e sobrepeso, evidenciadas pelo IMC, cerca de 42,0\% dos avaliados apresentaram eutrofia e 39,0\% sobrepeso. Esse achado pode ser explicado pela prevalência de sobrepeso em neoplasias de mama $(41,9 \%)$. Dados semelhantes à pesquisa realizada com 153 mulheres em tratamento oncológico na cidade de Brasília - DF mostraram prevalência de 36,6\% de sobrepeso na amostra estudada ${ }^{23}$. O estudo promovido por Aguilar Cordeiro et al. ${ }^{24}$ salienta a associação direta e significante entre o excesso de peso e a neoplasia de mama. Esses achados corroboram o estudo e podem ser explicados pelas alteraçóes do câncer de mama no organismo feminino, cujo ganho de peso é intensificado pela quimioterapia, pelo uso de medicamentos corticoides, ausência de atividade física, hábitos alimentares inadequados e pelo próprio processo de envelhecimento ${ }^{23}$.
Ao comparar o IMC com a ASG-PPP, observou-se que pacientes que apresentaram eutrofia, sobrepeso ou obesidade pelo IMC foram classificados como desnutridos moderados segundo a ASG-PPP. Porém, pelo IMC foi possível observar déficit nutricional em 18 indivíduos (10,8\%), enquanto, pela ASG-PPP, 45 pessoas $(21,1 \%)$ foram classificadas com desnutrição moderada. Um estudo realizado com 99 pessoas em tratamento quimioterápico em Minas Gerais demostrou 9,1\% desnutridos de acordo com o IMC, $37,4 \%$ desnutridos moderados e $31,3 \%$ gravemente desnutridos segundo a ASG-PPP ${ }^{25}$. Esses resultados apontam que a ASG-PPP é capaz de identificar com mais precisão os pacientes oncológicos desnutridos ou em risco de desnutrição do que o IMC. Portanto, o IMC não deve ser utilizado como único indicador de classificação nutricional do paciente oncológico, necessitando de avaliação antropométrica completa associada à ASG-PPP ${ }^{25}$.

Quanto à FPP, cerca de 55,0\% dos entrevistados apresentaram força adequada, corroborando os achados de Cavagnari et al. ${ }^{26}$, cuja FPP não indicou depleção na maioria dos casos avaliados. Já Limberger et al. ${ }^{6}$ identificaram diferença de força entre os gêneros e ainda salientam que o método de avaliar a força pode complementar a interpretação sobre a análise do estado nutricional dos pacientes.

Ao avaliar a funcionalidade por meio do ECOG, um estudo de Pereira et al. ${ }^{27}$ apontou que a maioria dos avaliados possuía algum comprometimento que limitava suas habilidades diárias (escores 3 e 4), o que vai de encontro com achados deste estudo, em que 69,0\% dos entrevistados apresentou escore 0 . Peixoto et al. ${ }^{28}$ demonstram em seu estudo que a maioria dos indivíduos se situou nos escores 0 e 1 , indicando nenhuma alteração funcional. Takahashi et al. ${ }^{29}$ avaliaram 264 pacientes com câncer em um hospital universitário do Japão, destes, $215(81,4 \%)$ apresentaram classificação do ECOG 0 e 1, corroborando os achados deste estudo.

Ressalta-se que este estudo apresentou algumas limitaçóes, como modelo de estudo transversal, heterogeneidade entre diagnósticos, ausência dos dados referentes ao estadiamento da doença e à topografia. Destaca-se também como uma limitação do estudo o viés de seleção da amostra, visto que foram selecionadas apenas as amostras que possuíam o questionário ECOG no prontuário.

Apesar das limitaçóes citadas, esta pesquisa apresentou pontos fortes, sendo composta por uma amostra significativa de indivíduos, sendo esses achados uma importante contribuição para a prática clínica do nutricionista e de outros profissionais da saúde. 


\section{CONCLUSÃO}

O estudo demonstrou que a maioria dos pacientes oncológicos em quimioterapia tinham satisfatório estado nutricional, adequada força muscular e eram totalmente ativos. De qualquer forma, entende-se que é imprescindível realizar diferentes métodos de avaliação nutricional e funcional, desde o início até a finalização do tratamento oncológico, tendo em vista os possíveis efeitos adversos.

Diante dos resultados apresentados, pode-se enfatizar a importância da avaliação e do acompanhamento nutricional adequado nesse público, sabendo-se que o estado nutricional debilitado é frequente em pacientes oncológicos e interfere de forma negativa nas habilidades de vida diárias do paciente.

\section{CONTRIBUIÇÕES}

Gabriela Argenta Isoton contribuiu na concepção e planejamento do estudo, obtenção, análise e interpretação dos dados e na redação. Caroline da Silva Scotti contribuiu na concepção e planejamento do estudo. Joana Zanotti contribuiu na concepçáo e planejamento do estudo, análise e interpretação dos dados e revisão crítica. Todas as autoras aprovaram a versão final a ser publicada.

\section{DECLARAÇÃO DE CONFLITO DE INTERESSES}

Nada a declarar.

\section{FONTES DE FINANCIAMENTO}

Não há.

\section{REFERÊNCIAS}

1. Instituto Nacional de Câncer José Alencar Gomes da Silva. ABC do câncer: abordagens básicas para o controle do câncer. 5. ed. rev. atual. ampl. Rio de Janeiro: INCA; 2019.

2. International Agency for Research on Cancer [Internet]. Lyon, France: IARC; [date unknown] - . Estimated agestandardized incidence rates (World) in 2018, all cancers, both sexes, all ages; [cited 2019 Apr 8]; [about 1 screen]. Available from: https://gco.iarc.fr/today/online-analysismap?Projectio $\mathrm{n}=$ globe.

3. Instituto Nacional de Câncer José Alencar Gomes da Silva. Estimativa 2020: incidência de câncer no Brasil [Internet]. Rio de Janeiro: INCA; 2019. [acesso 2020 abr. 27]. Disponível em: https://www.inca.gov.br/sites/ ufu.sti.inca.local/files//media/document//estimativa2020-incidencia-de-cancer-no-brasil.pdf
4. Gomes NS, Maio R. Avaliação subjetiva global produzida pelo próprio paciente e indicadores de risco nutricional no paciente oncológico em quimioterapia. Rev Bras Cancerol. 2015;61(3):235-242. doi: https:// doi.org/10.32635/2176-9745.RBC.2015v61n3.253

5. Silva SED, Costa JL, Araújo JS, et al. Os impactos da terapia quimioterápica e as implicaçóes para a manutençáo do cuidado: um estudo de representaçóes sociais. Rev Fundam Care. 2018;10(2):516-523. doi: http://dx.doi.org/10.9789/2175-5361.2018. v10i2.516-523

6. Limberger VR, Pastore CA, Abib RT. Associação entre dinamometria manual, estado nutricional e complicaçóes pós-operatórias em pacientes oncológicos. Rev Bras Cancerol. 2014;60(2):135-141. doi: https://doi. org/10.32635/2176-9745.RBC.2014v60n2.479

7. Carvalho ESV, Leão ACM, Bergmann A. Funcionalidade de pacientes com neoplasia gastrointestinal alta submetidos ao tratamento cirúrgico em fase hospitalar. ABCD, Arq Bras Cir Dig. 2018;31(1):1353. doi: https:// doi.org/10.1590/0102-672020180001e1353

8. Giacalone A, Quitadamo D, Zanet E, et al. Cancerrelated fatigue in the elderly. Support Care Cancer. 2013;21(10):2899-911. doi: https://doi.org/10.1007/ s00520-013-1897-1

9. Conselho Nacional de Saúde (BR). Resolução nº. 466, de 12 de dezembro de 2012. Diário Oficial da União, Brasília, DF; 2013 jun. 13. Seção 1, p. 59.

10. Gonzalez MC, Borges LR, Silveira DH, et al. Validação da versão em português da avaliaçáo subjetiva global produzida pelo paciente. Rev Bras Nutr Clin. 2010;25(2):102-8.

11. Ministério da Saúde (BR) [Internet]. Brasília, DF: Ministério da Saúde; 2017. IMC Adultos; [atualizado 2017 maio 30; acesso 2019 abr. 15]. Disponível em: http://www.saude.gov.br/component/content/ article/804-imc/40509-imc-em-adultos

12. Ministério da Saúde (BR) [Internet]. Brasília, DF: Ministério da Saúde; 2017. Avaliação do peso IMC na terceira idade; [atualizado 2017 maio 30; acesso 2019 abr. 15]. Disponível em: http://www.saude.gov.br/ component/content/article/804-imc/40511-avaliacaodo-peso-imc-na-terceira-idade

13. Oken MM, Creech RH, Tormey DC, et al. Toxicity and response criteria of the eastern cooperative oncology group. Am J Clin Oncol. 1982;5(6):649-55.

14. Cruz-Jentoft AJ, Bahat G, Bauer J, et al. Sarcopenia: revised european consensus on definition and diagnosis. Age Ageing. 2019;48(1):16-31. doi: https://doi. org/10.1093/ageing/afy169

15 Moura EC, Gomes M, Falcão MTC, et al. Desigualdades de gênero na mortalidade por causas externas no Brasil, 2010. Ciên Saúde Coletiva. 2015;20(3):779-788. doi: https://doi.org/10.1590/1413-81232015203.11172014 
16. Souza RG, Lopes TVC, Pereira SS, et al. Avaliação do estado nutricional, consumo alimentar e capacidade funcional em pacientes oncológicos. Braz J Oncol. 2017;13(44):1-11.

17. Instituto Nacional de Câncer José Alencar Gomes da Silva. Inquérito brasileiro de nutrição oncológica. Rio de Janeiro: INCA; 2013.

18. Cunha SFC, Tanaka LS, Salomão RG, et al. Nutritional screening in a university hospital: comparison between oncologic and non-oncologic patients. Food Nutr Sci. 2015;6(1):75-82. doi: http://dx.doi.org/10.4236/ fns.2015.61009

19. Lima TR, Silva DAS, Kovaleski DF, et al. Associação da força muscular com fatores sociodemográficos e estilo de vida em adultos e idosos jovens no Sul do Brasil. Ciênc Saúde Coletiva. 2018;23(11):3811-20. doi: https://doi. org/10.1590/1413-812320182311.27792016

20. Firnkes R, Pastore CA, Gonzalez MC. Influência do estado nutricional sobre a qualidade de vida em pacientes com cânceres de trato gastrintestinal e de pulmão préquimioterapia. Rev Bras Nutr Clin. 2014;29(1):26-30.

21. Carvalho ACLM, Martins PC, Araujo RB, et al. Parâmetros nutricionais em pacientes oncológicos atendidos em um centro de referência no sul de Minas Gerais, Brasil. Rev Bras Cancerol. 2018;64(2):159166. doi: https://doi.org/10.32635/2176-9745. RBC.2018v64n2.74

22. Silva NA, Pedraza DF, Menezes TN. Desempenho funcional e sua associação com variáveis antropométricas e de composição corporal em idosos. Ciênc Saúde Coletiva. 2015;20(12):3723-32. doi: https://doi. org/10.1590/1413-812320152012.01822015

23. Cordeiro ALO, Fortes RC. Estado nutricional e necessidade de intervenção nutricional em mulheres com câncer de mama em tratamento quimioterápico. Arq Catarin Med. 2015;44(4):96-108.

24. Aguilar Cordeiro MJ, Neri Sánchez M, Padilla López CA, et al. Sobrepeso/obesidad en mujeres y su implicación en el cáncer de mama; edad de diagnóstico. Nutr Hosp. 2012;27(5):1643-7. doi: https://doi.org/10.3305/ nh.2012.27.5.5998

25. Milani J, Pereira EMS, Barbosa MH, et al. Antropometria versus avaliação subjetiva nutricional no paciente oncológico. Acta Paul Enferm. 2018;31(3):240-6. doi: https://doi.org/10.1590/1982-0194201800035

26. Cavagnari MAV, Assis CF, Moreira GCRC, et al. Avaliação da qualidade de vida e do estado nutricional de pacientes oncológicos: parâmetros que se complementam? Rev HUPE. 2017;16(2):79-84. doi: https://doi. org/10.12957/rhupe.2017.37631

27. Pereira EEB, Santos NB, Sarges ESNF. Avaliação da capacidade funcional do paciente oncogeriátrico hospitalizado. Rev Pan-Amaz Saude. 2014;5(4):37-44. doi: http://dx.doi.org/10.5123/S2176-62232014000400005
28. Peixoto MI, Dourado KF, Andrade MIS, et al. Comparação entre diferentes métodos de triagem nutricional em pacientes oncológicos ambulatoriais. Nutr Clín Diet Hosp. 2017;37(3):35-43.

29. Takahashi M, Takahashi M, Komine K, et al. The G8 screening tool enhances prognostic value to ECOG performance status in elderly cancer patients: a retrospective, single institutional study. PLoS ONE. 2017;12(6):e0179694. doi: https://doi.org/10.1371/ journal.pone.0179694 